Published: March 15 $5^{\text {th }}, 2019$

Citation: Petz (2019) An Approach to the Cure of HIV using Cord Blood Hematopoietic Cell Transplantation. SPG BioMed 1(2).

Lawrence Petz M.D.

StemCyte Cord Blood Bank

Tel: 626 646-2502

Email:Ipetz@stemcyte.com

Funding: None.
COMMENTARY

\section{An Approach to the Cure of HIV using Cord Blood Hematopoietic Cell Transplantation}

Lawrence Petz M.D.

Author affiliation:

StemCyte Cord Blood Bank

HIV infection has already been cured by hematopoietic cell transplantation.

The cure was reported in the New England Journal of Medicine in 2009 by Dr. Gero Hütter and associates from Germany (1). The patient, Timothy Brown, had acute myeloid leukemia and HIV infection and is now referred to as "The Berlin Patient". It was well known at that time that HIV-1 enters host cells by binding to a CD4 receptor and then interacting with either CCR5 or the CXC chemokine receptor (CXCR4). Homozygosity for a 32-bp deletion (delta32/delta32; CCR5-/-) in the CCR5 allele results in an inactive CCR5 gene product and consequently confers high resistance against HIV-1 infection. Following transplantation using stem cells from an adult donor who had the CCR5-/- mutation, HIV could not be detected in Timothy Brown's peripheral blood, spinal fluid, lymph nodes, bone marrow, brain or Gl mucosa as assessed with RNA and proviral DNA PCR assays. Further, there has been no recurrence of HIV in more than 10 years. Thus, the "Berlin Patient" has had a functional cure if not a sterilizing cure of HIV.

\section{Copyright: (c) (i) ()}

(C) 2019 Science Publishing Group

This open access article is distributed under the terms of the Creative Commons Attribution Non-Commercial License. 


\section{Why have there been no further cures of HIV by hematopoietic cell transplantation?}

Since the hematopoietic cell transplant performed by Dr. Hütter cured HIV, the question arises as to why has this procedure not been repeated over and over and over again?

\section{a. Low incidence of CCR5-/- individuals and need for a very close HLA matched unit when adult donors are used.}

There are numerous reasons for the lack of repetition of the cure of HIV that was obtained by hematopoietic cell transplantation. The frequency of CCR5-/- donors is $<1 \%$ in Caucasians and much less in other ethnic groups. Patients who need a transplant usually have only a small number of available HLA-matched adult donors. Dr. Hütter's patient was very unusual in that he had 80 potential HLA-identical stem-cell donors, 62 of whom were tested and one was found to be CCR5/-. Indeed, investigators have tried to find CCR5-/- units from among available adult donors for transplantation of HIV-infected patients, but without success.

b. The need for very close HLA match when adult donors are used as source of stem cells for hematopoietic cell transplantation rather than cord blood stem cells.

It is quite difficult to find a CCR5-/- adult donor who is HLA matched with a patient since a very close HLA match between the donor cells and the patient is required to prevent serious adverse effects of the transplant. In marked contrast to adult donors, cord blood units require significantly less stringent HLA matching (2), and therefore an inventory of CCR5-/- cord blood units, when compared to an inventory of adult units, will provide a greatly improved probability of finding an adequately HLA-matched CCR5-/- donor for a patient who has HIV infection.

\section{c. Lack of confidence in cord blood transplantation.}

There are several reasons that cure of HIV using cord blood hematopoietic cell transplantation has not yet been accomplished. One of these is a misguided lack of confidence in the effectiveness of cord blood transplantation. Indeed, a recently completed clinical trial regarding transplantation of HIV infected patients included the following statement in the experimental protocol: "Allogeneic transplantation using cord blood unit(s) as a hematopoietic stem cell source is prohibited under this protocol" (3). However, more than 35,000 cord blood transplants have been performed and there is extensive data indicating the adequacy of cord blood transplantation compared to matched unrelated donors and even sibling donors $(2,4)$. Further, the incidence of chronic graft-versus-host disease (a major concern following hematopoietic cell transplantation) is significantly reduced in cord blood transplantation as compared to transplants using adult donors (2).

Two aspects of cord blood transplants that have been of concern are the difficulty of finding cord blood units with an adequate cell dose and the lengthy time to engraftment. These concerns are resolved by the use of combined haploidentical and cord blood (haplo/cord) transplantation (5). Using this procedure, the minimum cell dose of the cord blood unit is very reasonable at $1 \times 10(7)$ $\mathrm{TNC} / \mathrm{kg}$ and the median time to engraftment is only 11 days. The "winning unit" in haplo/cord transplants is almost always the cord blood and the haploidentical unit gradually disappears. 


\section{d. The expense of hematopoietic cell transplantation.}

Hematopoietic cell transplants are considered by some to be too expensive to be used as a technique to treat HIV patients. However, one must realize that the lifetime costs of persons who become infected with HIV at age 35 has been estimated to be $\$ 326,500$. Of the total cost. $60 \%$ is for antiretroviral medications, $15 \%$ for chronic disease medications, and $25 \%$ for non-medication costs (6). Also, from fiscal year 1981 through 2004, the US government invested over $\$ 150$ billion in combating the HIV/AIDS epidemic but no cures of HIV have resulted from such extraordinary expenditures. Moreover, the costs to society of an HIV infection extend well beyond the medical domain, including social service, housing, patient time, lost productivity and emotional distress to patients and their families.

\section{e. Complications of hematopoietic cell transplantation.}

Another commonly held opinion is that the morbidity and mortality rates associated with hematopoietic cell transplantation are too high to make the procedure useful for treatment of HIV-infected patients. Such opinions are largely derived from transplantation using stem cells from adult donors for transplantation of middle-aged or elderly patients with hematologic disorders who often have co-morbidities. In contrast, a study of 36 patients under the age of 50 who had advanced hematologic malignancies with a disease risk of low or intermediate resulted in a nonrelapse mortality of $8.3 \%$, and only one patient developed mild chronic graft-versus-host disease (personal communication, Dr. Koen van Besien).

If one selects for transplantation young HIV-infected patients with no co-morbidities, the adverse effects are significantly reduced. Since estimates indicate that there are about 37 million HIV infected patients in the world, finding significant numbers of young patients with no comorbidities can be expected to be no problem.

\section{f. Lack of availability of cord blood CCR5-/- units and lack of evidence that such units inhibit HIV infection.}

The question arises as to the availability of CCR5-/- cord blood units and whether there has been any experience in their use. An inventory of $>200$ CCR5-/- cord blood units has been established at StemCyte Cord Blood Bank in Baldwin Park, California after testing units provided by various cord blood banks in the U.S. as a well as StemCyte's own units. Also, an additional inventory of approximately the same size has been developed in Europe. Two hematopoietic cell transplants using cells from the StemCyte inventory have been performed and the results have been published $(7,8)$. Dr. Rafael Duarte in Barcelona transplanted a patient who had an aggressive lymphoma and HIV infection. The patient received a haplo/cord transplant using a CCR5-/- cord blood unit. Following transplantation, plasma HIV DNA load was undetectable by ultrasensitive analysis and upon cord blood full chimerism, the patient's peripheral blood cells were resistant to infection in vitro by the patient's viral isolate and by laboratory adapted HIV strains (7). Another CCR5-/- unit was inadvertently used as part of a double cord blood transplant for a patient with acute myelogenous leukemia who did not have HIV infection (8). The fact that one of the units used was CCR5-/- was determined only after transplantation had been undertaken. After engraftment, chimerism studies indicated that the "winning unit" was the CCR5-/- unit and the patient's peripheral cells were resistant to HIV infection in vitro. Unfortunately, both patients died as a result of a recurrence of their underlying hematologic malignancies several months following their transplant and this prevented definitive evaluation of a possible cure. Nevertheless, these data 
strongly support the use of cord blood as a platform for broader application of this curative technology to other HIV-infected individuals.

\section{g. Inability to use cord blood stem cell hematopoietic cell transplantation in a significant number of patients.}

It may seem that an inventory of several hundred cord blood units would not be of much significance in the cure of HIV infection. However, it should be noted that the National Marrow Donor Program has at least 296,000 cord blood units in its inventory (personal communication, Donna Regan). If a cure of HIV is obtained with a cord blood unit as a "proof of principle" certainly some of the billions of dollars expenditure by the U.S. Government in combating the HIV/AIDS epidemic could readily be transferred to expanding the inventory of CCR5-/- units available. Other sources of financial support would also likely be available to identify many more CCR5-/- units and thus provide a cure for a reasonably large number of patients.

\section{h. A rigidly held opinion that hematopoietic cell transplantation is too dangerous to be used as a cure of HIV infections.}

Although all of the above are significant, the major reason that cord blood units have not been used for cure of HIV infection is the rigidly held opinion in the U. S. that transplantation with CCR5-/- cells to cure HIV in patients who do not have an underlying indication for a transplant, such as leukemia or lymphoma, should not be performed. This opinion proposes that the adverse effects of hematopoietic cell transplantation are too severe, and antiretroviral drugs provide effective suppression of the clinical aspects of HIV infection even though it is known that there have been no cures of HIV.

Such a concept needs detailed scrutiny. One must consider the adverse effects of long-range HIV infection in the antiretroviral era. Published data (9) indicate that patients with long-term HIV infection even in this antiretroviral era have a risk of developing non-Hodgkin lymphoma that is 24.2 times greater than the general population. The risk of Hodgkin lymphoma is increased nearly 15 -fold greater than in non-infected individuals. Patients also remain at increased risk of acute leukemia, myelodysplastic syndromes, and cancers of the aeropharynx, lung, bladder and gastrointestinal tract (9). There is, however, speculation that modern antiretrovirals will prevent such adverse effects of long-term HIV infection although there are no published data or unpublished data to support such speculation. Also to be taken into account are data, reviewed above, which indicate that the adverse effects of haplo/cord blood transplants of young patients who have no co-morbidities using reduced intensity conditioning has far fewer significant side effects than has often been stated.

i. Ignoring the significance of stigma and individual patient's expressed desires.

Two further points are of major significance but are generally ignored. One point is the serious stigma associated with HIV infection. This can lead to severe depression and the impact on the quality of life can be dramatic (10). Indeed, published reports indicate that people living with HIV live in constant suffering, and many HIV infected patients feel that they have a new life characterized by the constant fear of stigmatism with serious consequences to individual and social development (11). Indeed, one such person stated, "It was the greatest impact I have ever experienced. I lost the joy from my life, and it was the most soul-wrenching thing that has ever 
happened to me" (11). It must also be noted that suicide rates are higher in HIV-infected patients than in the general population (12).

ii. The second point of major significance that seems to be universally ignored has been stated by Majhail et al (13): "Whether or not to proceed with transplantation in an individual patient is a clinical decision best made between the patient and his or her provider after a careful consideration of the alternatives, risks, and benefits of the procedure."

These two points are erroneously ignored perhaps because there is no physical evidence of either. Instead, if a patient feels that he or she would prefer the risk of a transplant with the possibility of a cure of HIV rather than the severe stigma and disruption of the quality of life posed by the HIV infection, such request would be denied by regulatory agencies. Thus, in regard to hematopoietic cell transplantation for cure of HIV infection, regulatory agencies do not respect individuals' preferences even if the patients are highly responsible and their conclusions are derived from detailed and well thought out considerations.

\section{An opinion based on the above.}

Regulatory agencies do not consider themselves responsible for the adverse effects of long-term HIV infection including deaths of HIV+ patients even though such agencies may prevent a possible cure and therefore require a young patient to suffer for many decades the long-term adverse effects of HIV infection, the extraordinary stigma, increased risk of Hodgkin's Disease, non-Hodgkin lymphoma, acute leukemia, myelodysplastic syndromes, and cancers of the aeropharynx, lung, bladder, gastrointestinal tract and cardiovascular disease as indicated above. Fully informed patients who have HIV infection but do not have an underlying indication for a transplant may prefer to take the risk of a transplant using CCR5-/- donor cells which have been shown to cure HIV. Such patients should be allowed to do so.

Unfortunately ........ that will not happen in the United States.

\section{References:}

1. Hütter G, Nowak D, Mossner M, et al. Long-term control of HIV by CCR5 Delta32/Delta32 stemcell transplantation. N Engl J Med 2009;360:692-8.

2. Barker JN, Kurtzberg J, Ballen K, et al. Optimal Practices in Unrelated Donor Cord Blood Transplantation for Hematologic Malignancies. Biol Blood Marrow Transplant 2017;23:882-96.

3. Alvarnas JC, Ambinder R. Protocol BTM CTN \#0903/AMC 080 HCT for Hematologic Cancers and Myelodysplastic Syndromes in HIV-Infected Patients.

4. Kurtzberg J. A History of Cord Blood Banking and Transplantation. Stem cells translational medicine 2017;6:1309-11.

5. van Besien K, Childs R. Haploidentical cord transplantation-The best of both worlds. Semin Hematol 2016;53:257-66. 
6. Schackman BR, Fleishman JA, Su AE, et al. The lifetime medical cost savings from preventing HIV in the United States. Medical care 2015;53:293-301.

7. Duarte RF, Salgado M, Sanchez-Ortega I, et al. CCR5 Delta32 homozygous cord blood allogeneic transplantation in a patient with HIV: a case report. The Lancet HIV 2015;2:e23642.

8. Petz LD, Burnett JC, Li H, et al. Progress toward curing HIV infection with hematopoietic cell transplantation. Stem cells and cloning : advances and applications 2015;8:109-16.

9. Alvarnas JC, Zaia JA, Forman SJ. How I treat patients with HIV-related hematological malignancies using hematopoietic cell transplantation. Blood 2017;130:1976-84.

10. Deeks SG. HIV infection, inflammation, immunosenescence, and aging. Annual Review of Medicine 2011;62:141-55.

11. LeWa-Moral JM, et al. Living with constant suffering: A different life following the diagnosis of HIV; J Assoc Nurses AIDS Care 2015;26:613-624.

12. Keiser O, Spoerri A, Brinkhof MW, et al. Suicide in HIV-infected individuals and the general population in Switzerland, 1988-2008. The American Journal of Psychiatry 2010;167:143-50.

13. Majhail NS, Farnia SH, Carpenter PA, et al. Indications for autologous and allogeneic hematopoietic cell transplantation: Guidelines from the American Society for Blood and Marrow Transplantation. Biol Blood Marrow Transplantation 2015;21:1863-1869. 